\title{
To achieve deep cuts in US emissions, state-driven policy is only slightly more expensive than nationally uniform policy
}

\begin{abstract}
Using a multi-sector model of human and natural systems, we find that the nationwide cost from state-varying climate policy in the United States is only one-tenth higher than that of nationally uniform policy. The benefits of state-led action - leadership, experimentation and the practical reality that states implement policy more reliably than the federal government - do not necessarily come with a high economic cost.
\end{abstract}

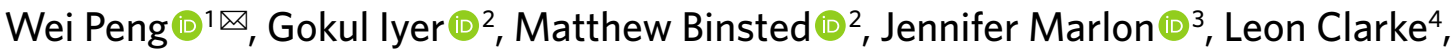 \\ James A. Edmonds (1) ${ }^{2}$ and David G. Victor ${ }^{5,6,7,8}$
}

BASED ON W. Peng et al. Nature Climate Change 11, 738-745 (2021).

\section{The policy problem}

Traditionally, assessment of the economics of climate policies has assumed that governments would implement idealized, optimal policies such as nationally uniform economy-wide carbon taxes. Yet over the last decade, actual policy experience has demonstrated that the reality is a lot more varied. Although some national governments are firmly leading climate policy, in much of the world, states, cities and other sub-national actors are leading the way. While uneven sub-national action is common, especially in large and politically diverse countries, little is known about its implications for costs. This question is particularly important for the United States, which is the world's second largest emitter and which has some of the highest variability in the world for sub-national action: while about 20 states aim to achieve net-zero electricity or economy-wide emissions around the middle of the century, many others are doing little or nothing, and their political leadership faces little sustained public pressure to strengthen policy action.

\section{The findings}

As compared to an idealized nationally uniform policy, we find that varying the state-level policy stringency by a factor of three will increase the nationwide cost by only about $10 \%$. Such results are robust under different national decarbonization targets, formulations for policy heterogeneity, and technology assumptions. The low cost hinges on two conditions. First is the availability of critical technologies (for example, low-carbon electricity and bioenergy with carbon capture and storage) and the ability to trade relevant energy products across state borders. Second is that there is at least some effort by every state. If a handful of states are not engaged at all, the leading states will need to adopt extremely expensive negative emissions technologies, which pushes up the nationwide cost. These two conditions could be difficult to meet in the real world given the technological, regulatory and political

\section{Messages for policy}

- Heterogeneous federal approaches to tackle climate change may be more sustainable politically; the extra cost, as compared with idealized uniform policy, may be low.

- Wealth, economic structure and carbon intensity vary by state; state-led policies can embrace regional differences in interests and capabilities, bringing large political benefits with small extra cost.

- Efforts to remove barriers for inter-state trade of energy products - electricity and liquid fuels, especially - could help to lower mitigation costs not only for the leading states but also nationally.

- Investing in critical technologies in harder-to-abate sectors could make it easier for jurisdictions that are lagging behind the leaders to adopt new technologies and policies.

- Deep emissions cuts hinge on nearly every state making some effort; strategies that elicit at least minimal effort from all while encouraging increased ambition and faster action from leading jurisdictions may be most effective.

realities. Future work should consider potential constraints on technology adoption as well as trade and state politics.

\section{The study}

We employed a highly-detailed process-based integrated assessment model (GCAM-USA) to examine the costs of state-driven climate action. GCAM-USA includes state-level representation of energy 


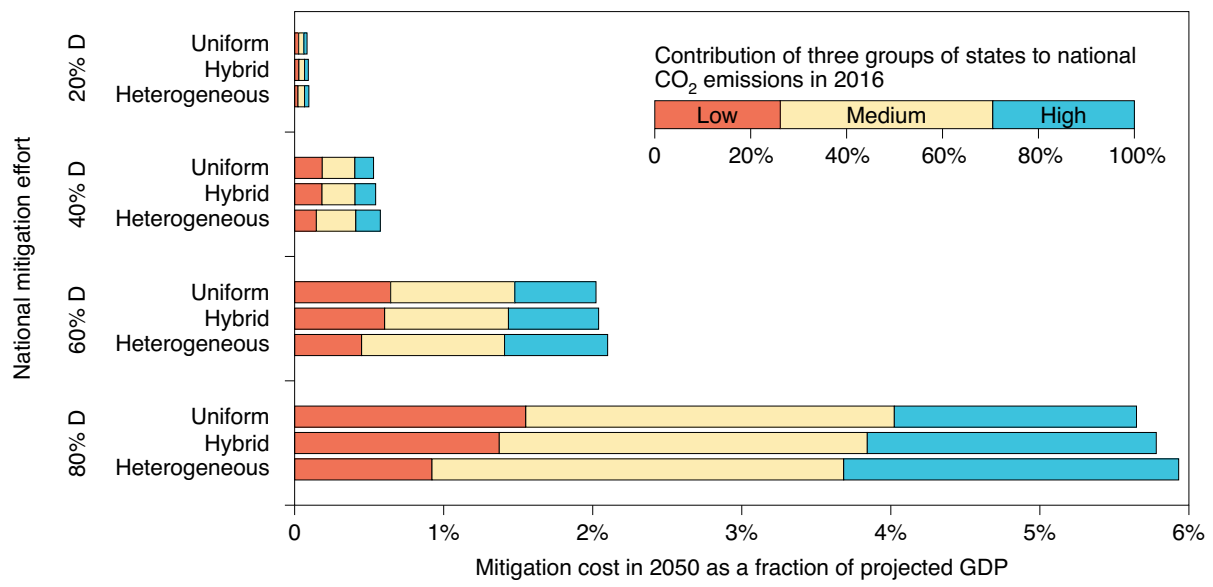

Fig. 1 Cost of state-driven and nationally uniform policy approaches to achieve varying decarbonization targets for the United States. Carbon mitigation cost in 2050 as a fraction of projected 2050 gross domestic product (GDP), by national mitigation effort (20-80\% decarbonization (D) by 2050 relative to 2005) and by subnational policy approach (uniform, hybrid and heterogeneous).

transformation and consumption sectors, as well as interactions between the economic, energy and land-use systems. These detailed representations allowed us to identify critical sectors, technologies and processes that determine the cost of heterogeneous policy efforts. Using this model, we examined the costs of 12 scenarios that vary across two dimensions: (1) national mitigation effort, measured according to four targets for cuts in national total greenhouse gas emissions, and (2) subnational policy approach, modelled as three degrees of heterogeneity in the stringency of state-level climate policy. We used surveys of public opinion in each state as a proxy for cross-state variation in policy stringency (Fig. 1). We further tested the effects of a wide range of alternative policy and technology assumptions on nationwide mitigation costs.

Published online: 28 October 2021

https://doi.org/10.1038/s41558-021-01193-5

Further reading

Iyer, G. et al. Measuring progress from nationally determined contributions to mid-century strategies. Nat. Clim. Change 7, 871-874 (2017).

This study utilized the same model (GCAM-USA) to examine the required transition in the energy system for the United States to achieve deep decarbonization by the middle of the century.

Peng, W. et al. Climate policy models need to get real about people - here's how. Nature 594, 174-176 (2021).
This commentary provides the broader context of the research agenda for bringing politics into integrated assessment models.

Keohane, R. O. \& Victor, D. G. Cooperation and discord in global climate policy. Nat. Clim. Change 6, 570-575 (2016).

This paper discussed decentralized policy strategy to tackle climate change, which could lead, incrementally, to deeper cooperation.

Sabel, C. F. \& Victor, D. G. Governing global problems under uncertainty: making bottom-up climate policy work. Clim. Change 144, 15-27 (2017).

This paper highlights the importance of institutional support for the success of bottom-up climate action.

Bauer, N. et al. Quantification of an efficiency-sovereignty trade-off in climate policy. Nature 588, 261-266 (2020).

While we studied non-uniform policy efforts across the US states, this study examined non-uniform efforts across countries using a global-scale integrated assessment model (REMIND-MAgPIE).

\section{Acknowledgements}

W.P. received a summer research stipend from the Penn State School of International Affairs. G.I., M.B. and J.A.E. received support from the Global Technology Strategy Program. D.G.V. draws funding, in part, from the Electric Power Research Institute, a non-profit R\&D organisation focused on the electric power sector. D.G.V. is also supported partly by donations to the Scripps Institution of Oceanography for research on emergency responses to climate change.

Competing interests

D.G.V. is a consultant to the shareholder group Engine No. 1. The other authors declare no competing interests. 